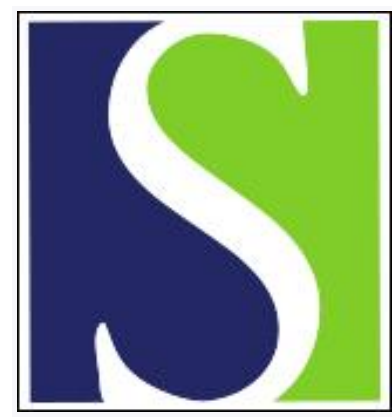

Scand J Work Environ Health 2014;40(3):215-229

https://doi.org/10.5271/sjweh.3420

Published online: 06 Mar 2014, Issue date: 01 May 2014

Occurrence of delayed-onset post-traumatic stress disorder: a systematic review and meta-analysis of prospective studies

by Utzon-Frank N, Breinegaard N, Bertelsen M, Borritz M, Eller NH, Nordentoft M, Olesen K, Rod NH, Rugulies R, Bonde JP

This review updates earlier work by including prospective studies published within the past five years and extends earlier work by inclusion of studies with late follow-up. Addition of some recent large studies to this review brings the combined number of participants in the meta-analysis to more than five times higher than in the latest review (Smid et al, 2009).

Affiliation: Department of Occupational and Environmental Medicine, Bispebjerg Bakke 23, Bispebjerg Hospital, Denmark. nicolai.utzon-frank.01@regionh.dk

Refers to the following texts of the Journal: 2013;39(4):325-334 2012;38(3):282-290

The following article refers to this text: $2020 ; 46(4): 339-349$

Key terms: accident; catastrophe; deployment; disaster; mental disease; meta-analysis; post-traumatic stress disorder; PTSD; review; systematic review; trajectory

This article in PubMed: www.ncbi.nlm.nih.gov/pubmed/24599261 


\title{
Occurrence of delayed-onset post-traumatic stress disorder: a systematic review and meta-analysis of prospective studies
}

by Nicolai Utzon-Frank, MD, ${ }^{1}$ Nina Breinegaard, MSc, PhD, ${ }^{1}$ Mette Bertelsen, MSc, PhD, ${ }^{2}$ Marianne Borritz, MD, PhD, ${ }^{1}$ Nanna Hurwitz Eller, MDSc, ${ }^{1}$ Merete Nordentoft, MD, PhD, ${ }^{3}$ Kasper Olesen, MSc, ${ }^{1,4}$ Naja Hulvej Rod, MSc, PhD, ${ }^{4}$ Reiner Rugulies, MSc, PhD, ${ }^{5}$ Jens Peter Bonde, MD, PhD ${ }^{1}$

Utzon-Frank N, Breinegaard N, Bertelsen M, Borritz M, Eller NH, Nordentoft M, Olesen K, Rod NH, Rugulies R, Bonde JP. Occurrence of delayed-onset post-traumatic stress disorder: a systematic review and meta-analysis of prospective studies. Scand J Work Environ Health. 2014;40(3):215-229. doi:10.5271/sjweh.3420

\begin{abstract}
Objective Post-traumatic stress disorder (PTSD) develops according to consensus criteria within the first 1-6 months after a horrifying traumatic event, but it is alleged that PTSD may develop later. The objective was to review the evidence addressing occurrence of PTSD with onset $>6$ months after a traumatic event (delayed-onset PTSD).

Methods Through a systematic search in PubMed, EMBASE, and PsycINFO, we identified 39 studies with prospective ascertainment of PTSD. A meta-analysis was performed in order to obtain a weighted estimate of the average proportion of delayed-onset PTSD cases, and meta-regression was used to examine effects of several characteristics.

Results Delayed-onset PTSD was reported in all studies except one, and the average prevalence across all follow-up time was 5.6\% [95\% confidence interval (95\% CI) $4.3-7.3 \%$ ]. The proportion with delayed-onset PTSD relative to all cases of PTSD was on average 24.5\% (95\% CI 19.5-30.3\%) with large variation across studies. In six studies with sub-threshold symptom data, delayed-onset PTSD seemed most likely an aggravation of early symptoms. The proportion with delayed-onset PTSD was almost twice as high among veterans and other professional groups compared to non-professional victims.

Conclusion Descriptive follow-up data suggest that PTSD may manifest itself $>6$ months after a traumatic event, delayed-onset PTSD most often, if not always, is preceded by sub-threshold PTSD symptoms, and a higher proportion of PTSD cases are delayed among professional groups. Contextual factors and biased recall may inflate reporting of PTSD and a cautious interpretation of prevalence rates seems prudent.
\end{abstract}

Key terms accident; catastrophe; deployment; disaster; mental disease; trajectory; PTSD.

Post-traumatic stress disorder (PTSD) is a mental disorder defined by intrusive recollections, avoidant behavior, and hyperarousal following the experience of a horrifying traumatic event. The PTSD diagnosis was introduced in the aftermath of the Vietnam War in the third edition of the American Diagnostic and Statistical Manual of Mental Disorders (DSM-III) [for a review, see (1)] . In Europe, the disorder was initially considered a phenomenon related to the Vietnam War and it took several years before the diagnosis was included in the World Health
Organization's International Classification of Diseases [(ICD-10 (2)]. A fourth disease criterion, social disability, was added to the DSM-IV criteria published in 1994 (3), but is not included in ICD-10 (2). The criteria will be revised in the ICD-11 (4).

PTSD is not only defined by specified symptoms but - unusually for any psychiatric disorder - also by its presumed cause: an unusual and horrifying psychological experience. The diagnostic entity has emerged based upon numerous follow-up studies of traumatized populations

1 Department of Occupational and Environmental Medicine, Bispebjerg University Hospital, Copenhagen, Denmark.

2 Danish Veteran Centre, Copenhagen, Denmark.

3 Psychiatric Centre Copenhagen, Bispebjerg University Hospital, Copenhagen, Denmark.

4 Department of Public Health, University of Copenhagen, Copenhagen, Denmark.

5 National Research Centre for the Working Environment, Copenhagen, Denmark.

Correspondence to: Nicolai Utzon-Frank, Department of Occupational and Environmental Medicine, Bispebjerg Bakke 23, Bispebjerg Hospital, Denmark. [E-mail: nicolai.utzon-frank.01@regionh.dk] 
(5), but the changing criteria over the past 30 years reflect ongoing discussions about the nature of the disorder (5). Since the vast majority of the voluminous PTSD literature is comprised of uncontrolled studies without appropriate reference groups, the causal link between the exposure (the horrifying traumatic event) and the outcome (the disease) is based upon the specificity of some of the symptoms and the apparent temporal link between symptoms and trauma. In DSM-III/IV and ICD-10, this is arbitrarily specified as 1-6 months after the triggering event.

The concept of delayed-onset PTSD was introduced in the original 1980 criteria, largely based on the impression that this was a common phenomenon amongst returning US Vietnam veterans, even though the evidence for this, as indeed for the rest of the criteria for PTSD, was lacking (6). Delayed-onset PTSD, diagnosed $>6$ months after the traumatic event or termination of a long-term exposure, has in particular been described among war veterans who develop symptoms during the years after returning home from war (7-12). Some of the PTSD symptoms such as disability (distress at a level causing impairment of social or occupational functioning), numbing and hyperarousal (sleep disturbance, irritability, hypervigilance, and cognitive problems) are non-specific complaints common in the general population. Therefore the lack of appropriate reference groups in descriptive studies of PTSD becomes a fundamental methodological issue - in particular as time from traumatic event to development of symptoms increases. This may partly explain why some researchers have doubted the existence of delayed-onset PTSD (13-15) and that delayed-onset PTSD has not yet been included in the International Classification of Mental Disease.

Determining the occurrence of delayed-onset PTSD has obvious implications for follow-up of trauma victims, treatment, and compensation. So far the issue of delayedonset PTSD has been addressed in two systematic reviews $(16,17)$. In 2007, Andrews et al (16)reviewed 10 casestudies and 19 studies with retro- or prospective data on the development of delayed-onset PTSD and emphasized the need to distinguish between (i) cases where PTSD symptoms start during the first months following a traumatic event, but the full PTSD syndrome fulfilling all DSM criteria only becomes manifest $>6$ months (exacerbated PTSD) and (ii) cases where the very first symptoms of PTSD start after a silent asymptomatic period of $\geq 6$ months with delayed development of the full PTSD syndrome (true delayed-onset PTSD). The authors concluded that the former commonly accounts for some $40 \%$ of military and $15 \%$ of civilian cases, while true delayedonset PTSD is rare. This conclusion is corroborated by a meta-analysis of 24 prospective studies with repeated examinations performed 1-6 months and $>1$ year after the traumatic event (17). Smid et al's review included papers published before April 2008 and observed delayed-onset
PTSD in $25 \%$ (95\% CI 23-27) of all PTSD cases, but few developed the disorder without early sub-threshold PTSD symptoms.

The present review updates earlier work by including prospective studies published within the past 5 years and extends earlier work by inclusion of prospective studies with late baseline examinations. Because of the inclusion of some recent large studies in our review, the combined number of participants in this meta-analysis is more than five times higher than in Smid et al's latest review (17). The objective of the present review was to estimate the proportion of delayed-onset PTSD and examine if it is associated with the type of traumatic event, type of population, and presence of sub-threshold PTSD symptoms during the initial months after the trauma.

\section{Methods}

\section{Eligibility criteria}

This review includes studies on adult populations exposed to horrifying traumatic events (ie, disasters, war zone conflicts, accidents, serious disease, or military combat). A comparison group of non-exposed was not requested. The outcome is the point prevalence of PTSD defined according to the DSM-III, DSM-IV, or ICD10 criteria. Only prospective studies with at $\geq 2$ PTSD assessments following the traumatic event(s) qualified for inclusion. We excluded studies with $<25$ participants because risk of recruitment bias was considered high.

\section{Literature search}

Eligible studies were identified through systematic searches in PubMed, EMBASE, and PsycINFO.

Only peer-reviewed, full, original papers published in English were accepted. Search steps and terms are detailed in figure 1. We first performed a restricted search covering the entire period 1 January 1980 through 30 March 2013 and then a broad search covering the past two years only. The restricted search only retrieved studies explicitly addressing delayed-onset PTSD while the broad search retrieved prospective follow-up studies in general (see figure 1 for details). Following this strategy, we identified 25 papers of which 4 were identified by the broad search. In addition, we found 14 papers from searching references in papers and reviews retrieved by the systematic search. A total of 39 studies were eligible for inclusion into the review. In 29 studies, the first baseline examination was performed $1-6$ months after the trauma or the end of a potentially traumatic period $(13,18-45)$ and in 10 studies, the baseline examination was performed $>6$ months after the trauma $(9,10$, 


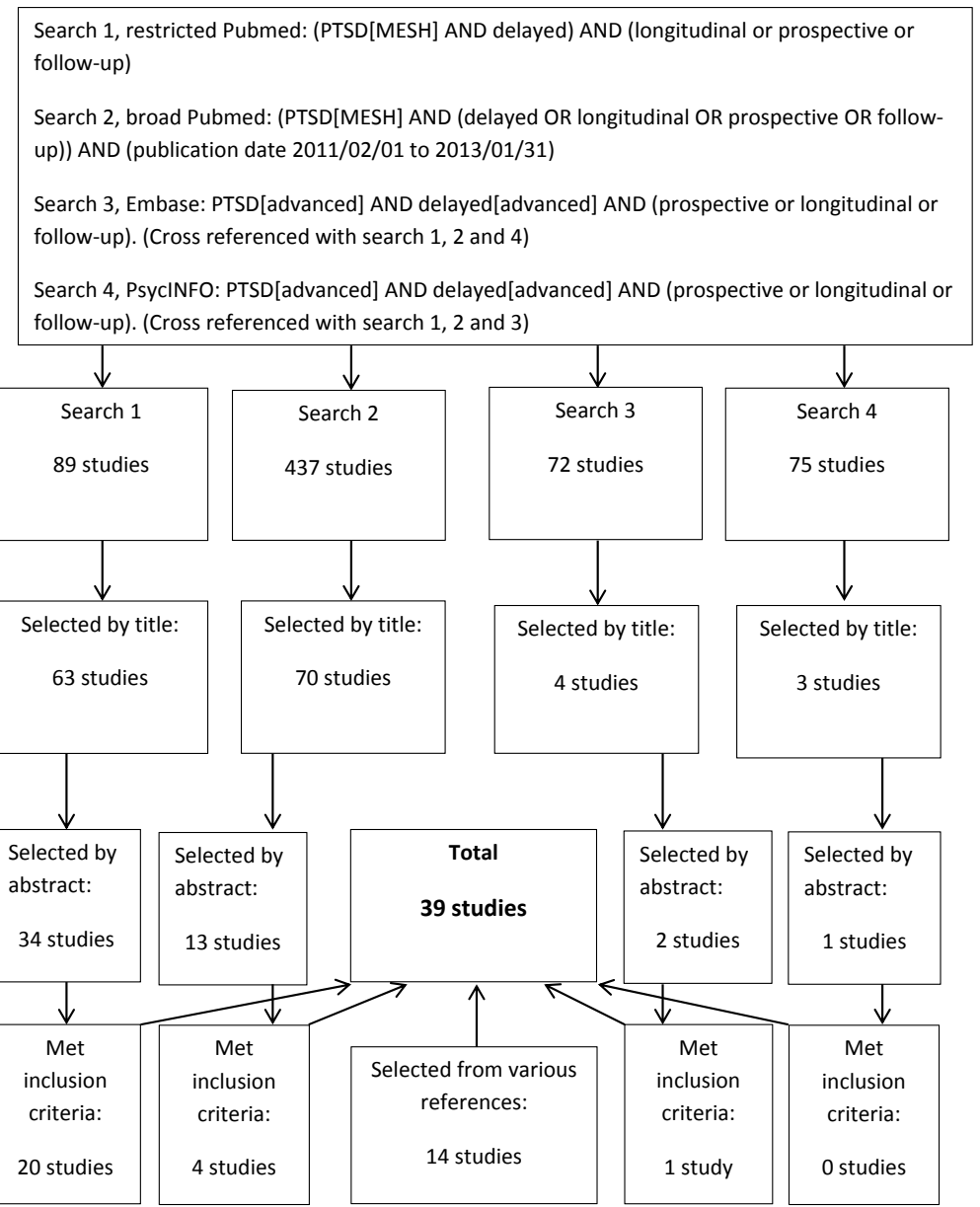

Figure 1. Flowchart showing the selection process of 39 studies with data on delayedonset post-traumatic stress disorder (PTSD).
46-53), see figure 2. Five studies included in the Smid et al (17) review were not included in the present study (two addressing children and adolescents, two with $<25$ participants and one in Polish), while three other studies published before 2009 were added $(21,25,28)$.

\section{Data extraction}

Two authors independently extracted data using a data extraction sheet containing the following information for each study: first author, country of origin, population characteristics, [age (mean, standard deviation, range), $\%$ male, $\%$ ethnic minority], trauma characteristics, PTSD diagnostic tool, number of assessments (months after event), and number of eligible, examined and diagnosed subjects.

\section{Definition of outcome}

We labeled PTSD diagnosed by telephone or face-toface interview by clinicians or trained interviewers as clinical PTSD. PTSD classified by self-reports based on the often-used PTSD checklist (PCL), a 17-item questionnaire, or similar instruments were labeled probable PTSD. PTSD symptoms based on self-reports with sum scores across all symptoms rather than the defining symptom-clusters were used to define possible PTSD. This latter approach was assumed to have the lowest specificity compared to the clinician-administered PTSD scale (CAPS), a psychiatric interview that is often used as the gold standard for PTSD diagnosis (55). If not otherwise specified, sub-threshold (or partial) PTSD defines individuals meeting the DSM-B criteria (intrusive memories) and either the $\mathrm{C}$ (avoidance and numbing) or D (hyperarousal) criteria - but not both. Finally, delayed-onset PTSD defines persons who develop the full PTSD syndrome according to DSM-III/IV or ICD10 criteria between a baseline assessment taking place after the traumatic event and a follow-up assessment taking place $\geq 6$ months later (figure 2 ).

\section{Quality assessment}

In order to provide a transparent evaluation of the epi- 


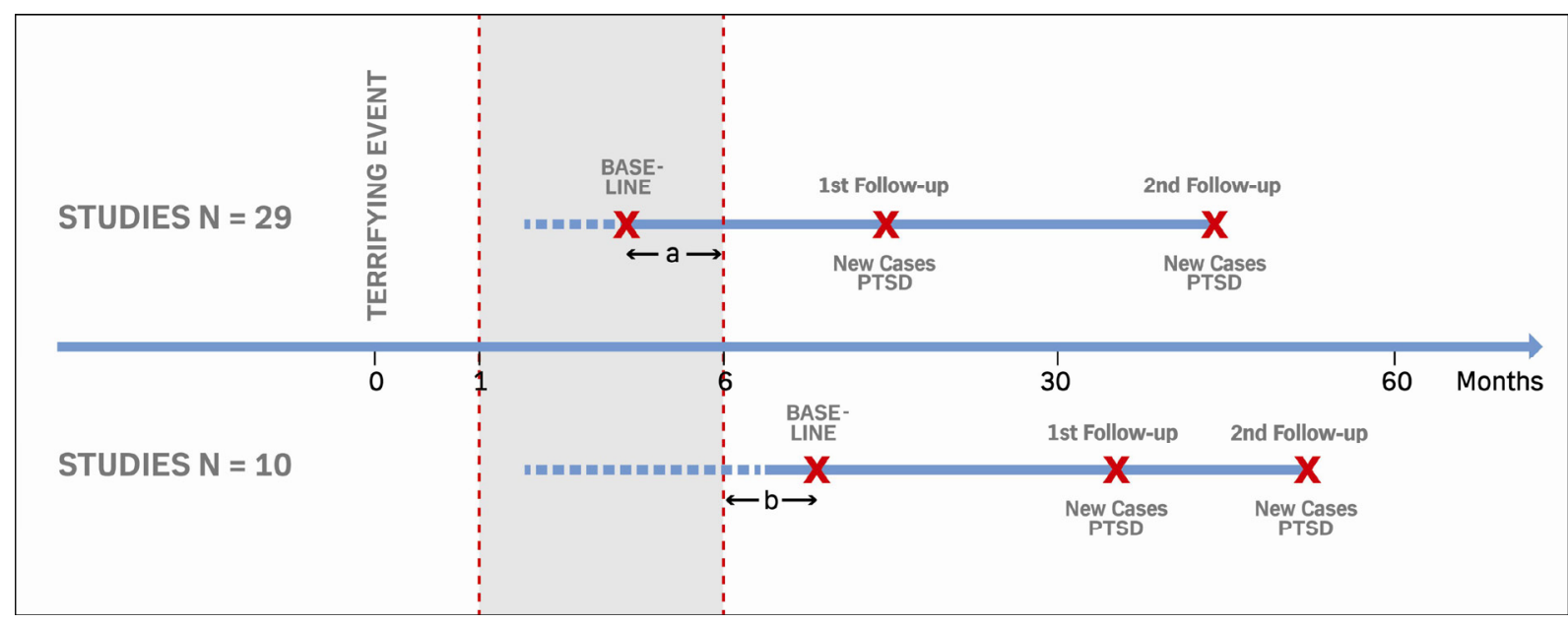

Figure 2. Timing of follow-up studies included in a systematic review of delayed-onset PTSD. A=duration of time between event and baseline

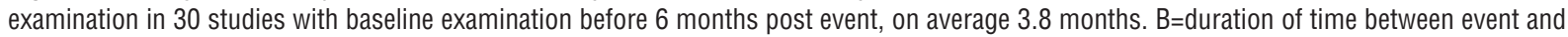
baseline examination in 10 studies with baseline examination before 6 months post event, on average 15.9 months

demiological evidence, we systematically assessed completeness of reporting and potential bias, respectively.

To assess completeness of reporting, we applied a modified version of van der Windt et al's (54) checklist, which has been used in several recent systematic reviews (55-57). This approach does not result in "quality" scores, which are discouraged in systematic reviews (58), but describes the degree to which a paper provides essential information on key study characteristics. Each publication was evaluated for completeness of reporting by an assessment of study design, sampling procedure, inclusion and exclusion criteria, response rates, description of the traumatic event, ascertainment of outcome, and statistics. We evaluated whether the paper provided essential details of each of these characteristics and assigned a value of 1 if the criterion was fulfilled and 0 if not. This evaluation is not an assessment of whether the various study characteristics (such as design, sampling etc) are more or less appropriate given the objective of this paper but rather the completeness of the provided information. Giving equal weight to each of the 7 characteristics, we considered completeness of reporting sufficient if the sum of the $0 / 1$ scores was $\geq 5$.

For assessment of bias we identified the most important threats to the validity of PTSD prevalence in prospective studies and distinguished between bias likely to result in inflated and deflated estimates, respectively (56). If participation in the baseline survey or subsequent follow-up surveys is dependent on presence of PTSDlike symptoms, PTSD prevalence rates are most likely biased towards too high values. We evaluated the risk of this recruitment and/or attrition bias by considering response rates (low primary response rate and/or high attrition related to likely bias). We did not identify bias that would systematically result in deflated prevalence rates. General limitations of studies mapping the population occurrence of PTSD are outlined in the discussion. Six authors evaluated the papers independently and at least two researchers evaluated each paper. Disagreements were resolved by the last author of this review.

\section{Data analysis}

The primary outcomes are the prevalence of PTSD and delayed-onset PTSD and the proportion of delayedonset PTSD relative to all identified cases of PTSD during rounds of follow-up. The prevalence of PTSD at each round of follow-up was calculated as the number of PTSD cases divided by all examined persons at that round. The prevalence of delayed-onset PTSD was defined as the number of persons fulfilling the PTSD criteria at one but not the preceding round of follow-up (new cases of PTSD since last examination) divided by all examined persons at that round. The proportion of delayed-onset PTSD was defined as the number of persons with delayed-onset PTSD divided by all PTSD cases identified during follow-up. To account for loss to follow up, we also computed an adjusted proportion of delayed-onset PTSD by multiplying observed cases by the inverse participation rate at follow-up. This approach assumes that loss to follow-up is independent of PTSD status. Studies with more than one follow-up examination contributed with two or more sets of PTSD estimates (prevalence of PTSD and delayed-onset PTSD and proportion of delayed-onset PTSD).

We computed the average prevalence of PTSD and delayed-onset PTSD and the proportion of delayed-onset PTSD across all studies using random effect meta-analyses, where the contribution from each study is weighted by the inverse variance. 
Individual study proportions are reported with exact binomial confidence intervals and pooled estimates were back-transformed to the original scale (59). One study (15) reported zero cases of delayed-onset PTSD and a continuity correction was applied by adding 0.5 to frequencies of events as well as non-events before logit transformation of proportions.

Heterogeneity between studies was evaluated by the $\mathrm{I}^{2}$ statistic, and funnel plots of the standard error against effect size was used to examine possible publication bias (60).

In sensitivity analyses, a meta-regression model was used to assess if the overall occurrence of delayed-onset PTSD depends on (i) low completeness of reporting and/ or likely bias, (ii) diagnostic criteria (clinical ascertainment, yes/no) and (iii) baseline examinations within 6 months (yes/no) (60).

In subgroup analyses, we present and compare estimates of delayed-onset PTSD in subgroups defined by the type of population (traumatized in relation to professional activity, yes/no) and by the type of trauma. The latter was defined by five trauma categories (natural disaster, terrorism, accident, injury/disease, and military combat/deployment). Moreover, we grouped studies into study populations with extended exposure and/or bereavement after the traumatic event (refugees, displaced after hurricanes, injured after accidents, serious disease) and study populations with less likelihood of persistent exposure after the initial traumatic event (not physically injured in accidents or terroristic acts and with unlikely loss of beloved ones, deployed soldiers). The mutually adjusted effects of these study-level variables on the proportion of delayed-onset PTSD were evaluated via meta-regression. Presence of sub-threshold symptoms during the initial post-traumatic period (yes/ no) was only reported in eight studies and therefore not included in meta-analyses.

All analyses were carried out in the R statistical software using functions metaprop and rma.uni in R-packages meta and metafor (61-63).

\section{Results}

\section{Characteristics of studies}

We included 39 prospective studies with a total of 30210 participants that provided $\geq 2$ point prevalences of PTSD after exposure to a traumatic event or following discontinuation of a potentially traumatic experience during military deployment (figure 1 and table 1). Only eight studies provided data on the presence of subthreshold PTSD symptoms during the initial six months $(9,14,18,19,28,37,48,53)$.
The majority of studies were performed in Europe and the United States and more studies have been undertaken in recent compared to earlier years (table 2). The average number of participants was 775 but 3 studies enrolled $>2500$ persons $(18,38,48)$. Exposure to terrorism was the traumatic event most often studied, followed by technological disasters and accidents, military deployment, injury (and disease), and natural disasters (table 2). The percentage of participants relative to all eligible at baseline was recorded in 24 studies with a median participation rate of $75 \%$ (range $5-100 \%$ ). The average loss to follow-up from one round to the next was $11 \%$ (range $0-35 \%$ ).

\section{Completeness of reporting and bias}

Completeness of reporting was considered high in 27 of the 39 studies (sum score 7/7 in 12 studies and 6/7 in 15 studies), but selection bias towards too high prevalence rates was likely in 18 studies because recruitment in the baseline examination and participation in followup rounds may not have been independent of PTSD symptoms $(23,25-28,30,32,34,37,40,42,44,45$, $47,49,51,52)$.

\section{Prevalence of PTSD and delayed-onset PTSD}

The overall weighted average baseline prevalence of (clinical, probable, and possible) PTSD was $19.7 \%$ [ $95 \%$ confidence interval $\left(95 \%\right.$ CI) $15.8-24.2 \%, \mathrm{I}^{2}=0.90$, $\mathrm{P}<0.001]$ with very large variation across studies (table 2 ) and the corresponding prevalence of delayed-onset PTSD was $5.6 \%\left(95 \%\right.$ CI $\left.4.3-7.3 \%, \mathrm{I}^{2}=0.91, \mathrm{P}<0.001\right)$.

\section{Proportion of delayed-onset PTSD}

The proportion of delayed-onset PTSD relative to all identified PTSD cases was on average $24.5 \%$ (95\% CI $\left.19.5-30.3 \%, \mathrm{I}^{2}=0.94, \mathrm{P}<0.001\right)$, with a wide dispersion across individual studies (table 3 , figure 3 ). Slightly higher rates were obtained in analyses accounting for loss to follow-up (weighted prevalence $25.9 \%$ (95\% CI $\left.20.6-32.0 \%, \mathrm{I}^{2}=0.94, \mathrm{P}<0.001\right)$ and a somewhat lower prevalence in an analysis restricted to the subset of 20 studies with complete reporting and less likely selection bias (weighted average $21.6 \%$ (95\% CI $15.8-28.8 \%$, $\left.\mathrm{I}^{2}=0.94, \mathrm{P}<0.001\right)$.

The proportion of delayed-onset PTSD was equivalent in studies with early (1-6 months after the trauma, $\mathrm{N}=29)$ and late ( $>9$ months after the trauma, $\mathrm{N}=10)$ baseline examination. The proportion of delayed-onset PTSD was non-significantly lower in the 15 studies applying clinical ascertainment of the PTSD diagnosis [weighted average $20.9 \%\left(95 \%\right.$ CI $\left.\left.15.9-27.2 \%, \mathrm{I}^{2}=0.68, \mathrm{P}<0.001\right)\right]$ in comparison with studies using questionnaire reports. 
Table 1. Characteristics of 39 studies on post-traumatic stress disorder (PTSD) included in a systematic review. [CAPS=Clinician-Administered PTSD Scale; DIS=Diagnostic Interview Schedule; DS=Disaster Supplement; DSM=Diagnostic and Statistical Manual of Mental Disorders; GHQ=General Health Questionnaire; IES=Impact of Event Scale; PCL=PTSD checklist; PSS=Post-traumatic Stress Symptom Scale; PTSD-I=PTSD inventory]

\begin{tabular}{|c|c|c|c|c|c|c|c|c|}
\hline Study & $\begin{array}{l}\text { Traumatic } \\
\text { event }\end{array}$ & Population & $\begin{array}{l}\text { Number of } \\
\text { partici- } \\
\text { pants } \\
(\mathrm{t} 1-\mathrm{t} 4)\end{array}$ & $\begin{array}{l}\text { Number of } \\
\text { assessments } \\
\text { (months since } \\
\text { trauma [t1-t4]) }\end{array}$ & Diagnostic tool & $\begin{array}{l}\text { Number } \\
\text { of PTSD } \\
(\mathrm{t} 1-\mathrm{t} 4)\end{array}$ & $\begin{array}{l}\text { Number of } \\
\text { delayed- } \\
\text { onset PTSD } \\
\text { (t1-t4) }\end{array}$ & $\begin{array}{l}\text { Proportion } \\
\text { of delayed- } \\
\text { onset PTSD }\end{array}$ \\
\hline \multicolumn{9}{|c|}{$\begin{array}{l}\text { Assessments }</>6 \\
\text { months after traumatic event }\end{array}$} \\
\hline $\begin{array}{l}\text { Berninger et al } \\
2010 \text {, USA (19) }\end{array}$ & $9 / 112001$ & $\begin{array}{l}\text { Firefighters } \\
\text { participating } \\
\text { in } 9 / 11\end{array}$ & $\begin{array}{l}5656 \\
5656\end{array}$ & $2(3,36)$ & $\begin{array}{l}\text { PCL- military } \\
\text { version }\end{array}$ & $\begin{array}{l}487 \\
628\end{array}$ & $391(t 2)$ & $44.5 \%$ \\
\hline $\begin{array}{l}\text { Blanchard et al } \\
1996, \text { USA (20) }\end{array}$ & $\begin{array}{l}\text { Motor vehicle } \\
\text { accident }\end{array}$ & $\begin{array}{l}\text { Motor vehicle } \\
\text { accident survivors }\end{array}$ & $\begin{array}{l}158 \\
145,132\end{array}$ & $3(3,6$ and 12$)$ & CAPS & $\begin{array}{l}48,23 \\
24\end{array}$ & $\begin{array}{l}2(\mathrm{t} 2), \\
4(\mathrm{t} 3)\end{array}$ & $11.1 \%$ \\
\hline $\begin{array}{l}\text { Bryant et al 2001, } \\
\text { Australia (21) }\end{array}$ & $\begin{array}{l}\text { Motor vehicle } \\
\text { accident }\end{array}$ & $\begin{array}{l}\text { Motor vehicle } \\
\text { accident survivors }\end{array}$ & $\begin{array}{l}171,134 \\
103\end{array}$ & $3(1,6,24)$ & $\begin{array}{l}\text { Composite } \\
\text { International } \\
\text { Diagnostic } \\
\text { Interview, PTSD } \\
\text { module }\end{array}$ & $\begin{array}{l}23 \\
\text { (6 month), } \\
25\end{array}$ & $5(\mathrm{t} 3)$ & $17.9 \%$ \\
\hline $\begin{array}{l}\text { Buckley et al } \\
\text { 1996, USA (22) }\end{array}$ & $\begin{array}{l}\text { Motor vehicle } \\
\text { accident }\end{array}$ & $\begin{array}{l}\text { Motor vehicle } \\
\text { accident survivors }\end{array}$ & $\begin{array}{l}158,158 \\
158\end{array}$ & $3(1,6$ and 12$)$ & CAPS & $62, \mathrm{Na}$ & $7(\mathrm{t} 3)$ & $10.1 \%$ \\
\hline $\begin{array}{l}\text { Carty et al 2006, } \\
\text { Australia (23) }\end{array}$ & Traumatic injury & $\begin{array}{l}\text { Traumatic injury } \\
\text { survivors }\end{array}$ & 301,301 & 2 (3 and 12) & CAPS & 23,30 & $14(\mathrm{t} 2)$ & $37.8 \%$ \\
\hline $\begin{array}{l}\text { Colville et al } \\
\text { 2012, UK (24) }\end{array}$ & $\begin{array}{l}\text { Child admitted in } \\
\text { hospital unit }\end{array}$ & $\begin{array}{l}\text { Primary carer of } \\
\text { admitted children in } \\
\text { hospital unit }\end{array}$ & 102,66 & 2 (3 and 12) & $\begin{array}{l}\text { Short form of } \\
\text { Davidson Trauma } \\
\text { Scale }\end{array}$ & 28,18 & $8(t 2)$ & $22.2 \%$ \\
\hline $\begin{array}{l}\text { Curran et al } \\
\text { 1990, Ireland } \\
(25)\end{array}$ & $\begin{array}{l}\text { Enniskillen } \\
\text { bombing } 1987\end{array}$ & Bombing survivors & 26,26 & 2 (6 and 12) & $\begin{array}{l}\text { GHQ and } \\
\text { psychiatric } \\
\text { interview }\end{array}$ & 13,10 & $2(\mathrm{t} 2)$ & $13.3 \%$ \\
\hline $\begin{array}{l}\text { Ehlers et al 1998, } \\
\text { UK }(26)\end{array}$ & $\begin{array}{l}\text { Motor vehicle } \\
\text { accident }\end{array}$ & $\begin{array}{l}\text { Motor vehicle acci- } \\
\text { dent survivors }\end{array}$ & $\begin{array}{l}1181 \\
888,781\end{array}$ & $3(1 / 2,3$ and 12$)$ & PSS & $\begin{array}{l}205(3 \\
\text { month), } \\
129\end{array}$ & $34(\mathrm{t} 3)$ & $14.2 \%$ \\
\hline $\begin{array}{l}\text { Gray et al } 2004, \\
\text { USA (27) }\end{array}$ & $\begin{array}{l}\text { US peacekeeping } \\
\text { mission in Somalia }\end{array}$ & $\begin{array}{l}\text { US veterans deployed } \\
\text { in Somalia }\end{array}$ & $\begin{array}{l}1040 \\
1040\end{array}$ & $2(41 / 2$ and 18$)$ & PCL & 70,115 & $68(\mathrm{t} 2)$ & $49.3 \%$ \\
\hline $\begin{array}{l}\text { Hauff et al 1994, } \\
\text { Norway (28) }\end{array}$ & Post-Vietnam war & $\begin{array}{l}\text { Vietnamese boat } \\
\text { refugees in Norway }\end{array}$ & 131,131 & 2 (3 and 36) & $\begin{array}{l}\text { Present State } \\
\text { Examination }\end{array}$ & 12,5 & $3(\mathrm{t} 2)$ & $20.0 \%$ \\
\hline $\begin{array}{l}\text { Hepp et al 2008, } \\
\text { Switzerland (29) }\end{array}$ & Severe injury & $\begin{array}{l}\text { Severe injury } \\
\text { survivor }\end{array}$ & $\begin{array}{l}121,90 \\
90,90\end{array}$ & $\begin{array}{l}4(1,6,12 \text { and } \\
36)\end{array}$ & CAPS & $5,3,2,4$ & $3(\mathrm{t} 4)$ & $37.5 \%$ \\
\hline $\begin{array}{l}\text { Jehel et al 2003, } \\
\text { France (30) }\end{array}$ & $\begin{array}{l}\text { Paris subway } \\
\text { bombing, } 1996\end{array}$ & Bombing survivors & 32,32 & 2 (6 and 32) & $\begin{array}{l}\text { Watson's PTSD-I } \\
+ \text { IES }\end{array}$ & 10,6 & $2(\mathrm{t} 2)$ & $16.7 \%$ \\
\hline $\begin{array}{l}\text { Johnson et al } \\
\text { 2002, USA (45) }\end{array}$ & $\begin{array}{l}\text { Clayton } \\
\text { courthouse } \\
\text { shooting, } 1992\end{array}$ & Shooting survivors & $\begin{array}{l}80,77, \\
77\end{array}$ & $3(2,12$ and 36$)$ & DIS/DS & 12,12 & $4(\mathrm{t} 4)$ & $25.0 \%$ \\
\hline $\begin{array}{l}\text { Kangas 2005, } \\
\text { Australia (31) }\end{array}$ & Cancer diagnosis & Cancer patients & $\begin{array}{l}82,63 \\
49\end{array}$ & $3(1,6$ and 12$)$ & CAPS & $\begin{array}{l}14(6 \\
\text { month), } 7\end{array}$ & $0(\mathrm{t} 3)$ & $0.0 \%$ \\
\hline $\begin{array}{l}\text { Karamustafalioglu } \\
\text { et al 2006, Turkey } \\
\text { (32) }\end{array}$ & $\begin{array}{l}\text { Earthquake in } \\
\text { Turkey, } 1999\end{array}$ & Surviving inhabitants & $\begin{array}{l}464,464, \\
464\end{array}$ & $3(2,8$ and 19$)$ & $\begin{array}{l}\text { PTSD self-test of } \\
\text { the Anxiety } \\
\text { Disorders } \\
\text { Association of } \\
\text { America }\end{array}$ & $\begin{array}{l}140,125 \\
49\end{array}$ & $\begin{array}{l}12(\mathrm{t} 2) \\
7(\mathrm{t} 3)\end{array}$ & $11.9 \%$ \\
\hline $\begin{array}{l}\text { Mayou et al 1997, } \\
\text { UK (34) }\end{array}$ & $\begin{array}{l}\text { Motor vehicle } \\
\text { accident }\end{array}$ & $\begin{array}{l}\text { Motor vehicle } \\
\text { accident survivors }\end{array}$ & $\begin{array}{l}188,171 \\
111\end{array}$ & $3(3,12$ and 60$)$ & $\begin{array}{l}\text { Questionnaire devel- } \\
\text { oped by Foa et al }\end{array}$ & $11,8,9$ & $\begin{array}{l}3(\mathrm{t} 2), \\
8(\mathrm{t} 3)\end{array}$ & $50.0 \%$ \\
\hline $\begin{array}{l}\text { Mayou et al 2001, } \\
\text { UK (35) }\end{array}$ & $\begin{array}{l}\text { Motor vehicle } \\
\text { accident }\end{array}$ & $\begin{array}{l}\text { Motor vehicle } \\
\text { accident survivors }\end{array}$ & $\begin{array}{l}1148 \\
865,773\end{array}$ & $3(0,3$ and 12$)$ & PSS & $\begin{array}{l}199 \text { (3 } \\
\text { month), } \\
131\end{array}$ & $39(\mathrm{t} 3)$ & $16.4 \%$ \\
\hline $\begin{array}{l}\text { McFarlane et al } \\
\text { 1988, Australia } \\
(46)\end{array}$ & $\begin{array}{l}\text { Bushfire disaster, } \\
1983\end{array}$ & Firefighters & 315,315 & $3(4,11$ and 29$)$ & $\mathrm{GHQ}$ & 77,93 & $\begin{array}{l}27(\mathrm{t} 2) \\
45(\mathrm{t} 3)\end{array}$ & $43.1 \%$ \\
\hline $\begin{array}{l}\text { Mollica et al } \\
\text { 2001, Croatia (36) }\end{array}$ & War in Bosnia & Bosnian refugees & 529,376 & 2 (1 and 36) & $\begin{array}{l}\text { Harvard Trauma } \\
\text { Questionnaire }\end{array}$ & 139,98 & $35(t 2)$ & $20.1 \%$ \\
\hline $\begin{array}{l}\text { North et al 1997, } \\
\text { USA (37) }\end{array}$ & $\begin{array}{l}\text { Mass shooting in- } \\
\text { cident, Texas } 1991\end{array}$ & Shooting survivors & 136,124 & 2 (2 and 14) & DIS/DS & 37,30 & $12(\mathrm{t} 2)$ & $24.5 \%$ \\
\hline $\begin{array}{l}\text { North et al 2004, } \\
\text { USA (14) }\end{array}$ & $\begin{array}{l}\text { Oklahoma City } \\
\text { bombing, } 1995\end{array}$ & Bombing survivors & 137,137 & 2 (6 and 17) & DIS/DS & 44,42 & $12(t 2)$ & $21.4 \%$ \\
\hline
\end{tabular}


Table 1. Continued

\begin{tabular}{|c|c|c|c|c|c|c|c|c|}
\hline Study & Traumatic event & Population & $\begin{array}{l}\text { Number of } \\
\text { participants } \\
\text { (t1-t4) }\end{array}$ & $\begin{array}{l}\text { Number of as- } \\
\text { sessments } \\
\text { (months since } \\
\text { trauma [t1-t4]) }\end{array}$ & Diagnostic tool & $\begin{array}{l}\text { PTSD (N) } \\
(\mathrm{t} 1-\mathrm{t} 4)\end{array}$ & $\begin{array}{l}\text { Number of } \\
\text { delayed- } \\
\text { onset PTSD } \\
(\mathrm{t} 1-\mathrm{t} 4)\end{array}$ & $\begin{array}{l}\text { Proportion } \\
\text { of delayed- } \\
\text { onset PTSD }\end{array}$ \\
\hline $\begin{array}{l}\text { O'Connor et al } \\
\text { 2010, Denmark } \\
\text { (38) }\end{array}$ & $\begin{array}{l}\text { Surgery for prima- } \\
\text { ry breast cancer }\end{array}$ & $\begin{array}{l}\text { Women with breast } \\
\text { cancer }\end{array}$ & $\begin{array}{l}3318 \\
2912\end{array}$ & 2 (3 and 15) & IES & 666,415 & $93(\mathrm{t} 2)$ & $21.4 \%$ \\
\hline $\begin{array}{l}\text { 0`Donnell et al } \\
\text { 2013, Australia } \\
\text { (15) }\end{array}$ & Traumatic injury & $\begin{array}{l}\text { Traumatic Injury } \\
\text { victims }\end{array}$ & 987,834 & 2 (3 and 15) & CAPS & 61,73 & 39 & $39.0 \%$ \\
\hline $\begin{array}{l}\text { Southwick et al } \\
1995, \text { USA (39) }\end{array}$ & $\begin{array}{l}\text { Operation Desert } \\
\text { Storm, } 1991\end{array}$ & $\begin{array}{l}\text { Deployed National } \\
\text { Guard reservists }\end{array}$ & $\begin{array}{l}62,62 \\
62\end{array}$ & $3(1,6$ and 24$)$ & $\begin{array}{l}\text { Mississippi scale } \\
\text { for combat-related } \\
\text { PTSD/ } \\
\text { DSM 3-R-based } \\
\text { PTSD symptom } \\
\text { scale }\end{array}$ & $4,4,8$ & $4(\mathrm{t} 2)$ & $50.0 \%$ \\
\hline $\begin{array}{l}\text { Stramrood } \\
\text { et al 2011, } \\
\text { Netherlands (40) }\end{array}$ & $\begin{array}{l}\text { Pregnancy } \\
\text { complications }\end{array}$ & Pregnant woman & 110,75 & $2(11 / 2$ and 15$)$ & $\begin{array}{l}\text { PSS self-report } \\
\text { questionnaire }\end{array}$ & 15,6 & $4(\mathrm{t} 2)$ & $21.1 \%$ \\
\hline $\begin{array}{l}\text { Su et al } 2010 \text {, } \\
\text { Taiwan (41) }\end{array}$ & $\begin{array}{l}\text { Taiwan Chi-Chi } \\
\text { earthquake, } 1999\end{array}$ & Earthquake survivors & $\begin{array}{l}2107 \\
1756\end{array}$ & $2(6$ and 36$)$ & $\begin{array}{l}\text { Disaster-related } \\
\text { Psychological } \\
\text { Screening Test }\end{array}$ & 418,78 & $40(\mathrm{t} 2)$ & $8.7 \%$ \\
\hline $\begin{array}{l}\text { Sungur et al } \\
\text { 2001, Turkey (44) }\end{array}$ & $\begin{array}{l}\text { "Sivas Disaster", } \\
1993\end{array}$ & Survivors & $\begin{array}{l}79,79 \\
79,79\end{array}$ & $\begin{array}{l}4(1,6,12 \text { and } \\
18)\end{array}$ & $\begin{array}{l}\text { PTSD interview } \\
\text { based on DSM 3-R } \\
\text { criteria }\end{array}$ & $\begin{array}{l}16,21 \\
22,26\end{array}$ & $13(\mathrm{t} 3)$ & $44.8 \%$ \\
\hline $\begin{array}{l}\text { Tjemsland et al } \\
\text { 1998, Norway } \\
\text { (42) }\end{array}$ & $\begin{array}{l}\text { Operable breast } \\
\text { cancer }\end{array}$ & $\begin{array}{l}\text { Women with breast } \\
\text { cancer }\end{array}$ & 106,106 & 2 (1 1/2 and 12) & $\mathrm{IES}+\mathrm{GHQ}$ & 15,13 & $6(\mathrm{t} 2)$ & $28.6 \%$ \\
\hline $\begin{array}{l}\text { Wadsworth et al } \\
2009, \text { USA (43) }\end{array}$ & $\begin{array}{l}\text { Hurricane Katrina, } \\
2005\end{array}$ & $\begin{array}{l}\text { Civilians rehoused } \\
\text { in Colorado }\end{array}$ & 93,72 & 2 (4 and 10) & UCLA PTSD index & 35,39 & $16(t 2)$ & $31.4 \%$ \\
\hline \multicolumn{9}{|c|}{$\begin{array}{l}\text { Assessments } \geq 6 \text { months } \\
\text { after traumatic event }\end{array}$} \\
\hline $\begin{array}{l}\text { Boscarino et al } \\
2009, \text { USA (63) }\end{array}$ & $9 / 112001$ & $\begin{array}{l}\text { New York City } \\
\text { adults }\end{array}$ & $\begin{array}{l}2368 \\
1681\end{array}$ & 2 (12 and 24) & $\begin{array}{l}\text { Diagnostic interview } \\
\text { (DSM-4) }\end{array}$ & 174,134 & $98(t 2)$ & $36.0 \%$ \\
\hline $\begin{array}{l}\text { Bowler et al 2011, } \\
\text { USA (48) }\end{array}$ & $9 / 112001$ & Police responders & $\begin{array}{l}2940 \\
2940\end{array}$ & 2 (30 and 66) & PCL & 230,485 & $329(\mathrm{t} 2)$ & $58.0 \%$ \\
\hline $\begin{array}{l}\text { Cukor et al 2011, } \\
\text { USA (58) }\end{array}$ & $9 / 112001$ & $\begin{array}{l}\text { Non-rescue } \\
\text { disaster workers }\end{array}$ & $\begin{array}{l}2960 \\
2960 \\
2960\end{array}$ & $\begin{array}{l}3(12,48 \text { and } \\
72)\end{array}$ & PCL & $\begin{array}{l}280,125 \\
48\end{array}$ & $\begin{array}{l}53(\mathrm{t} 2) \\
31(\mathrm{t})\end{array}$ & $14.6 \%$ \\
\hline $\begin{array}{l}\text { Eytan et al } 2010 \text {, } \\
\text { Kosovo (50) }\end{array}$ & War in Kosovo & $\begin{array}{l}\text { War exposed } \\
\text { civilians }\end{array}$ & 551,551 & 2 (24 and 96$)$ & $\begin{array}{l}\text { Mini } \\
\text { Neuropsychiatric } \\
\text { Interview (MINI) }\end{array}$ & 126,80 & $51(\mathrm{t} 2)$ & $28.8 \%$ \\
\hline $\begin{array}{l}\text { Goodwin et al } \\
2012 \text {, UK (10) }\end{array}$ & $\begin{array}{l}\text { War in Iraq } \\
2003\end{array}$ & $\begin{array}{l}\text { Deployed UK military } \\
\text { personnel }\end{array}$ & $\begin{array}{l}1397 \\
1397\end{array}$ & $2(24$ and 64$)$ & PCL & 57,63 & 44 (t2) & $43.6 \%$ \\
\hline $\begin{array}{l}\text { Harvey et al 2011, } \\
\text { UK (11) }\end{array}$ & $\begin{array}{l}\text { War in Iraq } \\
2003\end{array}$ & UK reservists & 552,552 & 2 (16 and 54) & PCL & 33,26 & 12 & $26.7 \%$ \\
\hline $\begin{array}{l}\text { Koren et al 2001, } \\
\text { Israel (51) }\end{array}$ & Traffic accident & Motorists & 74,58 & 2 (12 and 36$)$ & $\begin{array}{l}\text { Structured Clinical } \\
\text { interview (DSM-4) }\end{array}$ & 24,12 & $2(\mathrm{t} 2)$ & $7.7 \%$ \\
\hline $\begin{array}{l}\text { Scott et al 1995, } \\
\text { Scotland (52) }\end{array}$ & Lockerbie bombing & Citizens of Lockerbie & 25,25 & 2 (12 and 36) & $\begin{array}{l}\text { Structured clinical } \\
\text { interview (DSM } \\
3-\mathrm{R} \text { ) }\end{array}$ & 18,13 & $1(\mathrm{t} 2)$ & $5.3 \%$ \\
\hline $\begin{array}{l}\text { Solomon et al } \\
2006 \text {, Israel (53) }\end{array}$ & $\begin{array}{l}\text { Combat during the } \\
\text { Lebanon war } 1982\end{array}$ & Military veterans & $\begin{array}{l}214,214 \\
214,214\end{array}$ & $\begin{array}{l}4(12,24,36 \\
240)\end{array}$ & PTSD-I & $\begin{array}{l}96,95 \\
61,91\end{array}$ & $\begin{array}{l}24(t 2) \\
4(t 3) \\
13(t 4)\end{array}$ & $29.9 \%$ \\
\hline $\begin{array}{l}\text { Zhang et al 2013, } \\
\text { USA (61) }\end{array}$ & $\begin{array}{l}\text { US Embassy } \\
\text { bombing in } \\
\text { Nairobi } 1998\end{array}$ & $\begin{array}{l}\text { Directly exposed } \\
\text { Kenyan civilians }\end{array}$ & 128,128 & 2 (9 and 31) & DIS/DS & 52,36 & $11(\mathrm{t} 2)$ & $17.5 \%$ \\
\hline
\end{tabular}


Table 2. Characteristics of 39 original papers reporting the prevalence of newly onset post-traumatic stress disorder (PTSD) between $\geq 2$ post-trauma surveys.

\begin{tabular}{|c|c|c|c|c|c|c|}
\hline \multirow[t]{2}{*}{ Characteristic } & \multirow[t]{2}{*}{$\begin{array}{l}\text { Number of } \\
\text { studies }\end{array}$} & \multirow[t]{2}{*}{$\begin{array}{l}\text { Number of partici- } \\
\text { pants }^{\text {a }}\end{array}$} & \multirow[t]{2}{*}{$\%$ male (mean) } & \multirow[t]{2}{*}{$\begin{array}{l}\text { Average age, } \\
\text { years (mean) }\end{array}$} & \multicolumn{2}{|c|}{$\begin{array}{l}\text { Crude baseline PTSD } \\
\text { prevalence, } \%\end{array}$} \\
\hline & & & & & Mean & Min-max \\
\hline \multicolumn{7}{|l|}{ Publication year } \\
\hline Before 2000 & 11 & 2475 & 41.3 & 35.4 & 28 & $6-72$ \\
\hline $2000-<2008$ & 12 & 4271 & 59.5 & 37.8 & 22 & $7-45$ \\
\hline 2008-2013 & 16 & 23464 & 53.6 & 39.9 & 17 & $4-41$ \\
\hline \multicolumn{7}{|l|}{ Region } \\
\hline Europe & 16 & 9500 & 45.3 & 38.7 & 22 & $4-72$ \\
\hline USA & 12 & 15788 & 58.1 & 37.3 & 19 & $7-39$ \\
\hline Other & 11 & 4922 & 55.0 & 37.4 & 24 & $6-45$ \\
\hline \multicolumn{7}{|l|}{ Study size } \\
\hline $30-100$ & 10 & 642 & 50.9 & 39.5 & 31 & $6-72$ \\
\hline $101-500$ & 15 & 2834 & 41.7 & 34.5 & 24 & $2-64$ \\
\hline $501-3000$ & 12 & 17760 & 65.9 & 39.6 & 13 & $4-26$ \\
\hline$\geq 3001$ & 2 & 8974 & 50.0 & 46.8 & 14 & $9-20$ \\
\hline \multicolumn{7}{|l|}{ Type of trauma } \\
\hline Natural disaster & 4 & 2979 & 31.0 & 41.3 & 30 & $20-38$ \\
\hline Terrorism & 15 & 15772 & 55.9 & 38.7 & 26 & $7-72$ \\
\hline Accident & 10 & 4487 & 53,5 & 34.0 & 17 & $4-39$ \\
\hline Injury/disease & 5 & 3707 & 17,2 & 46.9 & 19 & $14-28$ \\
\hline Military combat/deployment & 5 & 3265 & 88.6 & 32.5 & 14 & $4-45$ \\
\hline \multicolumn{7}{|l|}{ Type of population } \\
\hline Citizens & 18 & 7285 & 47.1 & 39.1 & 30 & $7-72$ \\
\hline Patients & 11 & 7709 & 36.8 & 38.2 & 15 & $4-30$ \\
\hline Civil employees & 5 & 11951 & 66.2 & 38.7 & 14 & $8-30$ \\
\hline Military employees & 5 & 3265 & 88.6 & 32.5 & 14 & $4-45$ \\
\hline \multicolumn{7}{|l|}{ Diagnostic ascertainment } \\
\hline Clinical PTSD & 15 & 5833 & 52.5 & 37.5 & 24 & $4-72$ \\
\hline Probable PTSD & 15 & 17032 & 56.6 & 37.8 & 20 & $4-45$ \\
\hline PTSD symptoms & 9 & 7345 & 43.2 & 38.8 & 21 & $6-39$ \\
\hline
\end{tabular}

${ }^{a}$ Number of participants enrolled into the baseline survey (later than one month after the event).

In the subset of 10 studies with baseline examination $>6$ months after the traumatic event, the overall prevalence of PTSD declined with time from the traumatic event. Since the prevalence of delayed-onset PTSD was rather constant across time, the proportion of all identified PTSD cases with delayed-onset PTSD increased with time (table 4).

Determinants of the proportion of delayed-onset PTSD (subgroup analyses)

Type of population. The average proportion of delayedonset PTSD was significantly higher in populations of military personnel and professionals (firefighters, rescue workers, utility workers, and police) than among civilians. The weighted average was $37.6 \%$ (95\% CI $28.5-47.7 \%$, $\left.\mathrm{I}^{2}=0.94, \mathrm{P}<0.0001\right)$ among professionals and $20.3 \%(95 \%$ CI 16.4-24.9\%, $\left.\mathrm{I}^{2}=0.84, \mathrm{P}<0.001\right)$ among civilians.

Type of traumatic event. A comparison of the types of traumatic event (table 3 ) showed significant differences, with the group exposed to military combat/deployment having a dramatically higher average proportion of delayed-onset
PTSD cases compared to other trauma groups. All subgroup estimates are shown in the forest plot in figure 3 . The extended trauma group included 19 studies with 8363 participants and the non-extended group included 20 studies with 21847 participants. The weighted proportion of delayed-onset PTSD relative to all identified PTSD cases during follow-up was $21.5 \%\left(95 \%\right.$ CI $17.0-26.7, \mathrm{I}^{2}=0.78$, $\mathrm{P}<0.001$ ) in the group with persistent trauma and $27.7 \%$ $\left(95 \%\right.$ CI $\left.20.4-36.3, \mathrm{I}^{2}=0.96, \mathrm{P}<0.001\right)$ in the group without persistent trauma. This difference is non-significant.

A meta-regression model including all covariates related to methodology (baseline survey before or after six months, completeness of reporting, bias, and diagnostic tool), demographics and trauma characteristics (type of population and type of trauma) reduced heterogeneity, which, however, was still substantial $\left(\mathrm{I}^{2}=0.83\right.$, $\mathrm{P}<0.001)$. Funnel plot inspection did not lead to concerns about small studies systematically reporting a higher proportion of delayed-onset PTSD.

\section{Sub-threshold PTSD symptoms}

Only 8 of the 39 studies in this review provide informa- 
Table 3. Prevalence of post-traumatic stress disorder (PTSD) during follow-up in 39 follow-up studies of populations exposed to traumatic events. $[95 \% \mathrm{Cl}=95 \%$ confidence interval

\begin{tabular}{|c|c|c|c|c|c|c|}
\hline \multirow[t]{2}{*}{ Characteristic } & \multicolumn{3}{|c|}{ Number of PTSD cases } & \multicolumn{3}{|c|}{$\%$ Delayed-onset PTSD } \\
\hline & $\begin{array}{c}\text { Number of } \\
\text { participants }\end{array}$ & $A l^{b}$ & Delayed-onset ${ }^{c}$ & Mean d & $95 \% \mathrm{Cl}$ & Min-Max \\
\hline \multicolumn{7}{|l|}{ Publication year } \\
\hline Before 2000 & 2475 & 678 & 158 & 24.6 & $14-36$ & $5-50$ \\
\hline $2000-<2008$ & 4271 & 1038 & 252 & 24.6 & $16-33$ & $3-49$ \\
\hline 2008-2013 & 23464 & 3871 & 1172 & 27.3 & $20-35$ & $8-58$ \\
\hline \multicolumn{7}{|l|}{ Region } \\
\hline Europe & 9500 & 1900 & 348 & 23.5 & $17-30$ & $5-50$ \\
\hline USA & 15788 & 2486 & 995 & 31.3 & $21-42$ & $10-58$ \\
\hline Other & 4922 & 1201 & 239 & 22.7 & 13-33 & $3-45$ \\
\hline \multicolumn{7}{|l|}{ Study size, N1 } \\
\hline $30-100$ & 642 & 211 & 50 & 22.6 & $11-34$ & $3-50$ \\
\hline $101-500$ & 2834 & 919 & 228 & 25.1 & 18-32 & $10-50$ \\
\hline $501-3000$ & 17760 & 2820 & 820 & 28.6 & 19-38 & $9-58$ \\
\hline$\geq 3001$ & 8974 & 1637 & 484 & 28.4 & $0-23$ & $12-45$ \\
\hline \multicolumn{7}{|l|}{ Type of trauma } \\
\hline Natural disaster & 2979 & 835 & 147 & 23.8 & $0-50$ & $9-43$ \\
\hline Terrorism & 15772 & 2610 & 1012 & 22.9 & 13-33 & $8-50$ \\
\hline Accident & 4487 & 804 & 143 & 26.0 & 18-34 & $5-58$ \\
\hline Injury/disease & 3707 & 849 & 111 & 17.5 & $5-30$ & $3-29$ \\
\hline Military combat/deployment & 3265 & 429 & 169 & 39.9 & $26-54$ & $27-50$ \\
\hline \multicolumn{7}{|l|}{ Type of population } \\
\hline Citizens & 7285 & 1686 & 329 & 18.9 & $13-24$ & $3-45$ \\
\hline Patients & 7709 & 1516 & 240 & 25.2 & $17-34$ & $11-50$ \\
\hline Civil workers & 11951 & 1956 & 844 & 37.1 & $16-58$ & $15-58$ \\
\hline Military workers & 3265 & 429 & 169 & 39.9 & $26-54$ & $27-50$ \\
\hline \multicolumn{7}{|l|}{ Diagnostic ascertainment } \\
\hline Clinical PTSD & 5833 & 925 & 237 & 20.0 & 14-27 & $3-38$ \\
\hline Probable PTSD & 17032 & 2933 & 1063 & 29.2 & $22-37$ & $12-58$ \\
\hline PTSD symptoms & 7345 & 1729 & 282 & 29.4 & $16-43$ & $9-50$ \\
\hline
\end{tabular}

a Total number of participants in studies.

b Total number of PTSD cases diagnosed at baseline and all follow-up rounds.

c Total number of PTSD cases across follow-up rounds among individuals without full PTSD syndrome at the baseline examination.

${ }^{d}$ Average percentage of delayed-onset PTSD across studies [delayed cases/(cases at baseline+delayed cases)].

tion about sub-threshold symptoms during the initial post-traumatic period. First, firefighters in New York City had ongoing exposure to dead bodies and body parts several months after the collapse of the World Trade Center towers (18). In this professional group with extended exposure, only $8.2 \%(\mathrm{~N}=32)$ of all delayed-onset PTSD cases $(\mathrm{N}=391)$ did not have sub-threshold symptoms at the baseline examination taking place three months after the disaster. Second, a large survey of non-rescue disaster workers at NYC 9/11 (disaster recovery utility workers assisting in clean-up and utility service restoration) also reported few delayed-onset PTSD cases without initial sub-threshold PTSD symptoms (3 of 28 delayed-onset PTSD cases, 10.7\%) at earlier follow-up waves (48). Third, four smaller studies did not report any cases of delayed-onset PTSD without prior sub-threshold PTSD symptoms. Two of these addressed motor vehicle accident victims with 1-3 years of follow-up $(19,28)$, one study involved subjects involved in a mass shooting episode (37) and one addressed civilian employees that were exposed to the US Embassy bombing in Nairobi (53). Fourth, contrary to the above six studies, a large prospective cohort study of UK military personnel identified newly onset PTSD that developed during 40 months after the baseline examination, which was undertaken 6-60 months after return from deployment. Although the risk of delayed-onset PTSD was much higher among men with sub-threshold PTSD at the first examination in comparison with men without PTSD symptoms [odds ratio (OR) 10.2, 95\% CI 4.9-21.1, our calculation], 73\% $(\mathrm{N}=32)$ of all delayed-onset PTSD cases $(\mathrm{N}=44,3.5 \%$ of all 1397 participants) had no initial sub-threshold symptoms (9). Finally, an Australian study of 834 randomly selected patients with injuries, mostly following motor vehicle accidents reported that 22 of 39 patients $(65 \%)$ with delayed-onset PTSD after one year had minimal PTSD symptoms 3 months after the accident (39).

Severity of the traumatic exposure. Only four studies provided data on the risk of delayed-onset PTSD rela- 


\begin{tabular}{|c|c|c|c|c|c|c|}
\hline Study & Events & Total & & Proportion & $95 \% \mathrm{Cl}$ & Weight \\
\hline \multicolumn{7}{|l|}{ Event $=$ Natural disaster } \\
\hline Karamustafalioglu (2006) & 19 & 159 & $\mp$ & 0.119 & {$[0.074 ; 0.180]$} & $2.9 \%$ \\
\hline McFarlane (1988) & 72 & 167 & $\longrightarrow$ & 0.431 & {$[0.355 ; 0.510]$} & $3.1 \%$ \\
\hline Su (2010) & 40 & 458 & + & 0.087 & {$[0.063 ; 0.117]$} & $3.0 \%$ \\
\hline Wadsworth (2009) & 16 & 51 & & 0.314 & {$[0.191 ; 0.459]$} & $2.8 \%$ \\
\hline Random effects model & & 835 & & 0.205 & {$[0.078 ; 0.441]$} & $11.8 \%$ \\
\hline \multicolumn{7}{|c|}{ Heterogeneity: $I$-squared $=96.8 \%$, tau-squared $=1.249, p<0.0001$} \\
\hline \multicolumn{7}{|c|}{ Event $=$ Technological accident } \\
\hline Blancard (1996) & 6 & 54 & $\rightarrow$ & 0.111 & {$[0.042 ; 0.226]$} & $2.5 \%$ \\
\hline Bryant (2001) & 5 & 28 & $\longrightarrow$ & 0.179 & {$[0.061 ; 0.369]$} & $2.4 \%$ \\
\hline Buckley (1996) & 7 & 69 & + & 0.101 & {$[0.042 ; 0.198]$} & $2.6 \%$ \\
\hline Carty (2006) & 14 & 37 & & 0.378 & {$[0.225 ; 0.552]$} & $2.7 \%$ \\
\hline Ehlers (1998) & 34 & 239 & $\mp$ & 0.142 & {$[0.101 ; 0.193]$} & $3.0 \%$ \\
\hline Hepp (2008) & 3 & 8 & & 0.375 & {$[0.085 ; 0.755]$} & $1.8 \%$ \\
\hline Koren (2001) & 2 & 26 & + & 0.077 & {$[0.009 ; 0.251]$} & $1.8 \%$ \\
\hline Mayou (1997) & 11 & 22 & & 0.500 & {$[0.282 ; 0.718]$} & $2.5 \%$ \\
\hline Mayou (2001) & 39 & 238 & + & 0.164 & {$[0.119 ; 0.217]$} & $3.0 \%$ \\
\hline O'Donnell (2013) & 22 & 83 & & 0.265 & {$[0.174 ; 0.373]$} & $2.9 \%$ \\
\hline Random effects model & & 804 & & 0.203 & {$[0.143 ; 0.280]$} & $25.2 \%$ \\
\hline \multicolumn{7}{|c|}{ Heterogeneity: I-squared $=75.1 \%$, tau-squared $=0.3005, p<0.0001$} \\
\hline \multicolumn{7}{|c|}{ Event $=$ Terrorism and shooting } \\
\hline Berninger (2010) & 391 & 878 & $\mp$ & 0.445 & {$[0.412 ; 0.479]$} & $3.1 \%$ \\
\hline Boscarino (2009) & 98 & 272 & & 0.360 & {$[0.303 ; 0.420]$} & $3.1 \%$ \\
\hline Bowler (2011) & 329 & 567 & $\mp$ & 0.580 & {$[0.538 ; 0.621]$} & $3.1 \%$ \\
\hline Cukor (2011) & 48 & 328 & $\rightarrow$ & 0.146 & {$[0.110 ; 0.189]$} & $3.1 \%$ \\
\hline Curran (1990) & 2 & 15 & & 0.133 & {$[0.017 ; 0.405]$} & $1.7 \%$ \\
\hline Eytan (2010) & 51 & 177 & & 0.288 & {$[0.223 ; 0.361]$} & $3.0 \%$ \\
\hline Hauff (1994) & 3 & 15 & & 0.200 & {$[0.043 ; 0.481]$} & $2.0 \%$ \\
\hline Jehel (2003) & 2 & 12 & $\rightarrow$ & 0.167 & {$[0.021 ; 0.484]$} & $1.7 \%$ \\
\hline Johnson (2002) & 4 & 16 & & 0.250 & {$[0.073 ; 0.524]$} & $2.2 \%$ \\
\hline Mollica (2001) & 35 & 174 & $\rightarrow$ & 0.201 & {$[0.144 ; 0.268]$} & $3.0 \%$ \\
\hline North (1997) & 12 & 49 & & 0.245 & {$[0.133 ; 0.389]$} & $2.7 \%$ \\
\hline North (2004) & 12 & 56 & - & 0.214 & {$[0.116 ; 0.344]$} & $2.8 \%$ \\
\hline Scott (1995) & 1 & 19 & & 0.053 & {$[0.001 ; 0.260]$} & $1.3 \%$ \\
\hline Sungul (2001) & 13 & 29 & & 0.448 & {$[0.264 ; 0.643]$} & $2.6 \%$ \\
\hline Zhang (2013) & 11 & 63 & $\longrightarrow$ & 0.175 & {$[0.091 ; 0.291]$} & $2.7 \%$ \\
\hline Random effects model & & 2670 & & 0.265 & {$[0.193 ; 0.352]$} & $38.2 \%$ \\
\hline \multicolumn{7}{|c|}{ Heterogeneity: $I$-squared $=94 \%$, tau-squared $=0.5038, p<0.0001$} \\
\hline \multicolumn{7}{|l|}{ Event $=$ Traumatic injury } \\
\hline Colville (2012) & 8 & 36 & - & 0.222 & {$[0.101 ; 0.392]$} & $2.6 \%$ \\
\hline Kangas (2005) & 0 & 14 & 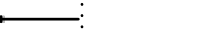 & 0.000 & {$[0.000 ; 0.232]$} & $0.8 \%$ \\
\hline O'Connor (2010) & 93 & 759 & + & 0.123 & {$[0.100 ; 0.148]$} & $3.1 \%$ \\
\hline Stramrood (2011) & 4 & 19 & & 0.211 & {$[0.061 ; 0.456]$} & $2.2 \%$ \\
\hline Tjemsland (1998) & 6 & 21 & & 0.286 & {$[0.113 ; 0.522]$} & $2.4 \%$ \\
\hline Random effects model & & 849 & $<$ & 0.175 & {$[0.108 ; 0.270]$} & $11.1 \%$ \\
\hline \multicolumn{7}{|c|}{ Heterogeneity: I-squared $=56 \%$, tau-squared $=0.1995, p=0.0589$} \\
\hline \multicolumn{7}{|c|}{ Event $=$ Military deployment } \\
\hline Goodwin (2012) & 44 & 101 & $\mp$ & 0.436 & {$[0.337 ; 0.538]$} & $3.0 \%$ \\
\hline Gray (2004) & 68 & 138 & $\longrightarrow$ & 0.493 & {$[0.407 ; 0.579]$} & $3.0 \%$ \\
\hline Harvey (2011) & 12 & 45 & & 0.267 & {$[0.146 ; 0.419]$} & $2.7 \%$ \\
\hline Solomon (2006) & 41 & 137 & $\div$ & 0.299 & {$[0.224 ; 0.383]$} & $3.0 \%$ \\
\hline Southwick (1995) & 4 & 8 & & 0.500 & {$[0.157 ; 0.843]$} & $1.9 \%$ \\
\hline \multirow{2}{*}{\multicolumn{7}{|c|}{$\begin{array}{l}\text { Random effects model } \\
\text { Heterogeneity: } I \text {-squared }=72.7 \% \text {, tau-squared }=0.1495, p=0.0054\end{array}$}} \\
\hline & & & & & & \\
\hline \multirow{3}{*}{\multicolumn{3}{|c|}{$\begin{array}{l}\text { Random effects model } 5587 \\
\text { Heterogeneity: } I \text {-squared }=94.3 \% \text {, tau-squared }=0.713, p<0.0001\end{array}$}} & & 0.242 & {$[0.193 ; 0.301]$} & $100 \%$ \\
\hline & & & & & & \\
\hline & & & 0.5 & 1 & & \\
\hline
\end{tabular}

Figure 3. Forest plot displaying randomeffects meta-analysis of point estimates of the proportion of delayedonset post-traumatic stress disorder (PTSD) by type of traumatic event [ $95 \% \quad \mathrm{Cl}=95 \%$ confidence interval]. tive to indicators of the severity of the traumatic exposure and highly heterogeneous definitions of exposure preclude meta-analysis. Here we provide descriptive information from these studies. Among firefighters, time of arrival at the World Trade Center (WTC) scene after the 9/11 attacks was related to risk of developing PTSD including delayed-onset PTSD (18). Those firefighters that arrived first are assumed to experience the most intense fear and horror and had the highest risk of delayed-onset PTSD. In a large UK cohort of military personnel, events related to combat exposure ("in a combat role during deployment", "thought might be killed", "discharged weapon") and history of depression predicted development of delayed-onset PTSD (as well as normal-onset PTSD), while relationship breakdown and leaving the military did not (9). In this study, the baseline examination took place up to 5 years after returning from deployment. In a random sample of 
Table 4. Prevalence of post-traumatic stress disorder (PTSD) a and absolute ${ }^{b}$ and relative ${ }^{c}$ prevalence of delayed-onset PTSD according to duration from trauma to baseline examination. [95\% $\mathrm{Cl}=95 \%$ confidence interval]

\begin{tabular}{|c|c|c|c|c|c|}
\hline \multirow{2}{*}{$\begin{array}{l}\text { Time after } \\
\text { traumatic } \\
\text { event } \\
\text { (months) }\end{array}$} & \multirow[t]{2}{*}{$\begin{array}{l}\text { Number of } \\
\text { participants }\end{array}$} & \multicolumn{2}{|c|}{$\begin{array}{c}\text { PTSD } \\
\text { prevalence (\%) }\end{array}$} & \multicolumn{2}{|c|}{$\begin{array}{l}\text { Proportion delayed- } \\
\text { onset PTSD }(\%)\end{array}$} \\
\hline & & All & Newly onset & Mean a & $95 \% \mathrm{Cl}$ \\
\hline $0-6$ & 17853 & 27.0 & 6.8 & 25.6 & $20-31$ \\
\hline $7-12$ & 5769 & 41.2 & 7.2 & 18.5 & $6-31$ \\
\hline $13-24$ & 2891 & 15.8 & 4.9 & 33.0 & $10-56$ \\
\hline$>24$ & 2940 & 19.3 & 11.2 & 58.0 & \\
\hline
\end{tabular}

${ }^{a}$ All identified PTSD cases relative to number of participants at baseline.

${ }^{\mathrm{b}}$ All PTSD cases with delayed-onset PTSD relative to number of participants at baseline.

c Delayed-onset PTSD cases relative to all PTSD cases.

adults living in New York City on 9/11 2001, the number of WTC-related events was related to the risk of PTSD, but not to the risk of delayed-onset PTSD (64). Finally, Sungur and Kaya (43) observed a higher prevalence of delayed-onset PTSD among survivors saved from a burning hotel in Sivas, Turkey, compared to two other groups assumed to experience less traumatic exposure. This study only included 79 participants (43).

\section{Discussion}

This systematic review updates and extends a previous meta-analysis (17) by almost doubling the number of included papers and including five times more participants. In particular, several large longitudinal and population-based studies of US and UK military personnel and of citizens and professionals exposed to the $9 / 11$ terror attacks adds to this new knowledge base $(9,18,47,48,64)$. Across all 39 prospective studies encompassing some 30000 participants, the occurrence of delayed-onset PTSD was ascertained by interview or questionnaire in $7 \%$ at some timepoint during followup and among some $25 \%$ of these cases the onset was delayed $\geq 6$ months.

\section{Comparison with the literature}

Our results corroborate findings in an earlier review based upon group- as well as case-reports (16) and a systematic review applying the same methodology as ours (17). Military personnel, firefighters, rescue workers, and police tended to have a lower absolute prevalence of PTSD, but a significantly higher proportion of delayedonset PTSD in comparison with civilian groups. Also this result is consistent with the reviews by Andrews et al (16) and Smid et al (17), but it has not been reported earlier that rescue workers and other professionals apparently share a higher proportion of delayed-onset PTSD with military men.

The prevalence and even the existence of delayedonset PTSD has been much discussed - partly because of different definitions of PTSD (16). According to Andrews et al, delayed-onset PTSD is rare in the absence of sub-threshold symptoms during the initial months after the traumatic event but rather frequent if some PTSD symptoms were present (16). If so, delayedonset PTSD is best regarded as an exacerbation or progression of subclinical PTSD. Findings in our review do not provide unequivocal support for this statement. Delayed-onset PTSD was preceded by subsyndromal symptoms in $>90 \%$ of cases in six studies $(18,19,28$, $37,48,53)$, but two recent large studies indicate more frequent occurrence of PTSD without prior symptoms $(9,39)$. However, sub-threshold PTSD in the UK study was defined by a PCL score of 40-49 on a scale from 17-85 (10). Obviously a lower cut-off level would result in fewer cases of delayed-onset PTSD without prior symptoms. Moreover, the study by O'Donnell included social impairment in the criteria for sub-syndromal PTSD. Thus the reporting of delayed-onset PTSD without preceding symptoms in these studies may be related to use of more strict criteria for sub-threshold PTSD.

\section{Strengths}

Our review benefits from a large number of high-quality prospective studies with outcome definitions that adhere to the DSM-III and IV criteria. No studies applied the slightly different ICD criteria. The prospective data collection entirely excludes bias introduced by retrospective recall of symptom onset. This is important because recall of earlier symptoms seems to be poor. For example, the majority of survivors of a mass shooting episode, who recovered from PTSD diagnosed 1-2 months after the shooting, did not recall PTSD symptoms one year later (37).

\section{Limitations}

Since studies were heterogeneous with respect to PTSD occurrence, we choose to report delayed-onset PTSD as a proportion of the overall prevalence of PTSD as suggested by others (17). In spite of this standardized measure of delayed-onset PTSD occurrence, the variation between and within subgroups defined by type of traumatic event was high. Characteristics related to study methodology (such as timing of surveys, length of followup, and diagnostic tools) do not seem to be important while demographic factors and type of populations do.

Attrition is a concern in follow-up studies. A Dutch longitudinal study of a large group of fire-disaster survivors with follow-up 14 years after the disaster did 
not indicate differences among responders and nonresponders with respect to the severity of the disaster experience, but Dutch survivors with mental health problems were less likely to participate in follow-up surveys while the opposite was found among immigrants (65). Furthermore, soldiers with sub-threshold PTSD symptoms may be less likely to deploy in new missions (66), which may bias studies that only include participants deployed several times (9). Thus healthrelated selective attrition seems likely.

Since only few studies performed an assessment at exactly six months after the traumatic event, this approach is associated with less accurate information on the timing of onset with potential inflation of prevalence rates. Similarly, the prevalence of delayed-onset PTSD recorded in the ten studies with baseline examination after nine months or more may be deflated to the extent a time-limited first episode PTSD had subsided before the baseline examination. In such cases, the apparent delayed-onset PTSD represents retriggering of a disorder with early onset. However, rates of delayed-onset PTSD were not lower in the ten studies with baseline examination after six months.

Unspecific PTSD symptoms such as insomnia, anxiety, and restlessness are common and may be present in the population before the traumatic event. Often used diagnostic tools such as the symptom checklist do not take this into account, which results in inflated estimates of PTSD occurrence.

\section{Causal inference}

The studies reviewed in this paper do not provide unequivocal evidence that disasters and other traumatic events are causally linked to delayed psychiatric morbidity including PTSD. This is because all studies are observational without appropriate reference groups of unexposed persons raising concern about biased results due to contextual factors. It has been shown that the aim and setting of a study may strongly influence the reporting and attribution of subjective symptoms (67) and descriptive studies of PTSD inherently point to the assumed causes. As early as 1983, LaGuardia et al (68) raised concern that studies reporting high rates of post-war delayed-onset stress disorders among Vietnam veterans might reflect the researchers' predisposition towards pathological interpretation and use of questionnaires biased in a direction suggesting maladjustment. In an experimental study of 38 randomly selected veterans, they showed that priming (biasing of the response profile of an individual to favor one direction of responding over another) by a positive, neutral, or negative introductory text, respectively, significantly influenced the subsequent questionnaire responses. They concluded that uncontrolled studies of veterans with focus on symptoms and maladjustment were methodologically flawed by selection and expectation bias (68).

Although inflated estimates of delayed-onset PTSD occurrence in descriptive and uncontrolled studies with focus on pathology seem inevitable (69-71), there are also arguments supporting causal links between traumatic events and occurrence of delayed-onset PTSD. First, longitudinal studies of PTSD symptom trajectories following traumatic events provide evidence of time links between exposure and development of symptoms (15). Slow development of symptoms following a severe psychological trauma seems more reliably related to the trauma than symptoms that develop after a long period without any symptoms. Second, some studies show an exposure-response relationship with higher risk for delayed-onset PTSD among persons with more severe traumatic exposure $(9,18,43)$. Third, in one study of military personnel, sociodemographic and health changes during the follow-up period (such as leaving the army or divorce) did not predict development of delayed-onset PTSD and thus are not competing or alternative risk factors (9). However, the statistical power to evaluate the latter was limited and there is a lack of studies that explore competing risk factors during the post-trauma follow-up period.

\section{Professionals versus civilians}

It is a notable observation that the proportion of delayedonset PTSD cases was almost twice as high among professional groups such as veterans, firefighters, rescue workers, utility workers, and police compared to civilians exposed to traumatic events. And some data indicate that military personnel are at higher risk of developing PTSD after a latent period with few symptoms than persons in other settings (72). Soldiers and other hierarchical professional groups may be reluctant to report symptoms until they become severe because of their fears of a negative impact on career and/or negative responses from their peers (72). Economic compensation systems and a need for a medical diagnosis to be granted sickness absence or social disability benefits may also influence reporting of symptoms (9). On the contrary, it is fully accepted that citizens react with fear and horror when involved in traumatic events. For the same reason professionals with early-onset symptoms might be less likely to participate in surveys. Thus, reporting and selection bias may in part explain delayed-onset PTSD without apparent initial symptoms in professional groups.

\section{Concluding remarks}

Descriptive follow-up data indicate that PTSD may manifest itself $>6$ months after a traumatic event, most 
often with sub-threshold PTSD symptoms bridging the traumatic event and disorder onset. The proportion of delayed-onset PTSD seems substantially higher among military personnel and other professionals. The unspecificity of some PTSD symptoms, the uncontrolled design of the majority of studies, and the likely impact on reporting of contextual factors such as media attention, study setting, objectives, and recruitment procedures calls for a cautious interpretation of causal relationships.

\section{Acknowledgment}

The study was supported by a grant from the Danish Work Environment Research Found.

The authors declare no conflicts of interest.

\section{References}

1. Jones E, Wessely S. A paradigm shift in the conceptualization of psychological trauma in the 20th century. J Anxiety Disord. 2007;21:164-75. http://dx.doi.org/10.1016/j. janxdis.2006.09.009.

2. WHO. The ICD-10 Classification of Mental and Behavioral Disorders. Geneva, Switzerland: WHO; 1992.

3. American Psychiatry Association. Diagnostic and statistical manual of mental disorders. 4th ed. Arlington: American Psychiatric Publishing Inc; 1994.

4. Maercker A, Brewin CR, Bryant RA, Cloitre M, Reed GM, van $\mathrm{OM}$, et al. Proposals for mental disorders specifically associated with stress in the International Classification of Diseases-11. Lancet. 2013;381:1683-5. http://dx.doi. org/10.1016/S0140-6736(12)62191-6.

5. Galea S, Nandi A, Vlahov D. The epidemiology of posttraumatic stress disorder after disasters. Epidemiol Rev. 2005;27:78-91.

6. Wessely S, Jones E. Psychiatry and the Lessons of Vietnam: What were they and are they still relevant? War and Society. 2004;22:89-103. http://dx.doi. org/10.1179/072924704791202130.

7. Jones M, Sundin J, Goodwin L, Hull L, Fear NT, Wessely S, et al. What explains post-traumatic stress disorder (PTSD) in UK service personnel: deployment or something else? Psychol Med. 2013;43(8):1703-12. http://dx.doi.org/10.1017/ S0033291712002619.

8. Pinder RJ, Greenberg N, Boyko EJ, Gackstetter GD, Hooper TI, Murphy D, et al. Profile of two cohorts: UK and US prospective studies of military health. Int J Epidemiol. 2012;41:1272-82. http://dx.doi.org/10.1093/ije/dyr096.

9. Goodwin L, Jones M, Rona RJ, Sundin J, Wessely S, Fear NT. Prevalence of delayed-onset posttraumatic stress disorder in military personnel: is there evidence for this disorder?: Results of a prospective UK cohort study. J Nerv

Ment Dis. 2012;200:429-37. http://dx.doi.org/10.1097/ NMD.0b013e31825322fe.

10. Harvey SB, Hatch SL, Jones M, Hull L, Jones N, Greenberg $\mathrm{N}$, et al. The long-term consequences of military deployment: a 5-year cohort study of United kingdom reservists deployed to Iraq in 2003. Am J Epidemiol. 2012;176:1177-84. http:// dx.doi.org/10.1093/aje/kws248.

11. Hotopf M, Hull L, Fear NT, Browne T, Horn O, Iversen A, et al. The health of UK military personnel who deployed to the 2003 Iraq war: a cohort study. Lancet. 2006;367:1731-41. http://dx.doi.org/10.1016/S0140-6736(06)68662-5.

12. Ismail K, Blatchley N, Hotopf M, Hull L, Palmer I, Unwin C, et al. Occupational risk factors for ill health in Gulf veterans of the United Kingdom. J Epidemiol Community Health. 2000;54:834-8. http://dx.doi.org/10.1136/jech.54.11.834.

13. North CS, Kawasaki A, Spitznagel EL, Hong BA. The course of PTSD, major depression, substance abuse, and somatization after a natural disaster. J Nerv Ment Dis. 2004;192:823-9. http://dx.doi.org/10.1097/01.nmd.0000146911.52616.22.

14. O’Donnell ML, Varker T, Creamer M, Fletcher S, McFarlane AC, Silove D, et al. Exploration of delayedonset posttraumatic stress disorder after severe injury. Psychosom Med. 2013;75:68-75. http://dx.doi.org/10.1097/ PSY.0b013e3182761e8b.

15. Breslau N, Reboussin BA, Anthony JC, Storr CL. The structure of posttraumatic stress disorder: latent class analysis in 2 community samples. Arch Gen Psychiatry. 2005;62:1343-51. http://dx.doi.org/10.1001/archpsyc.62.12.1343.

16. Andrews B, Brewin CR, Philpott R, Stewart L. Delayed-onset posttraumatic stress disorder: a systematic review of the evidence. Am J Psychiatry. 2007;164:1319-26. http://dx.doi. org/10.1176/appi.ajp.2007.06091491.

17. Smid GE, Mooren TT, van der Mast RC, Gersons BP, Kleber RJ. Delayed posttraumatic stress disorder: systematic review, meta-analysis, and meta-regression analysis of prospective studies. J Clin Psychiatry. 2009;70:1572-82. http://dx.doi. org/10.4088/JCP.08r04484.

18. Berninger A, Webber MP, Niles JK, Gustave J, Lee R, Cohen $\mathrm{HW}$, et al. Longitudinal study of probable post-traumatic stress disorder in firefighters exposed to the World Trade Center disaster. Am J Ind Med. 2010;53:1177-85. http://dx.doi. org/10.1002/ajim.20894

19. Blanchard EB, Hickling EJ, Barton KA, Taylor AE, Loos WR, Jones-Alexander J. One-year prospective follow-up of motor vehicle accident victims. Behav Res Ther. 1996;34:775-86. http://dx.doi.org/10.1016/0005-7967(96)00038-1.

20. Bryant RA, Harvey AG. Delayed-onset posttraumatic stress disorder: a prospective evaluation. Aust N Z J Psychiatry. 2002;36:205-9. http://dx.doi.org/10.1046/j.14401614.2002.01009.x

21. Buckley TC, Blanchard EB, Hickling EJ. A prospective examination of delayed onset PTSD secondary to motor vehicle accidents. J Abnorm Psychol. 1996;105:617-25. http:// dx.doi.org/10.1037/0021-843X.105.4.617.

22. Colville G, Pierce C. Patterns of post-traumatic stress 
symptoms in families after paediatric intensive care. Intensive Care Med. 2012;38:1523-31. http://dx.doi.org/10.1007/ s00134-012-2612-2.

23. Carty J, O'Donnell ML, Creamer M. Delayed-onset PTSD: a prospective study of injury survivors. J Affect Disord. 2006;90:257-61. http://dx.doi.org/10.1016/j. jad.2005.11.011.

24. Curran PS, Bell P, Murray A, Loughrey G, Roddy R, Rocke LG. Psychological consequences of the Enniskillen bombing. Br J Psychiatry. 1990;156:479-82.:479-82.

25. Ehlers A, Mayou RA, Bryant B. Psychological predictors of chronic posttraumatic stress disorder after motor vehicle accidents. J Abnorm Psychol. 1998;107:508-19. http://dx.doi. org/10.1037/0021-843X.107.3.508.

26. Gray MJ, Bolton EE, Litz BT. A longitudinal analysis of PTSD symptom course: delayed-onset PTSD in Somalia peacekeepers. J Consult Clin Psychol. 2004;72:909-13. http:// dx.doi.org/10.1037/0022-006X.72.5.909.

27. Hauff E, Vaglum P. Chronic posttraumatic stress disorder in Vietnamese refugees. A prospective community study of prevalence, course, psychopathology, and stressors. J Nerv Ment Dis. 1994;182:85-90. http://dx.doi. org/10.1097/00005053-199402000-00004.

28. Hepp U, Moergeli H, Buchi S, Bruchhaus-Steinert H, Kraemer B, Sensky T, et al. Post-traumatic stress disorder in serious accidental injury: 3-year follow-up study. $\mathrm{Br} \mathrm{J}$ Psychiatry. 2008;192:376-83. http://dx.doi.org/10.1192/bjp. bp.106.030569.

29. Jehel L, Paterniti S, Brunet A, Duchet C, Guelfi JD. Prediction of the occurrence and intensity of post-traumatic stress disorder in victims 32 months after bomb attack. Eur Psychiatry. 2003;18:172-6. http://dx.doi.org/10.1016/S09249338(03)00043-9.

30. Johnson SD, North CS, Smith EM. Psychiatric disorders among victims of a courthouse shooting spree: a three-year follow-up study. Community Ment Health J. 2002;38:181-94. http://dx.doi.org/10.1023/A:1015269521969.

31. Kangas M, Henry JL, Bryant RA. The course of psychological disorders in the 1st year after cancer diagnosis. J Consult Clin Psychol. 2005;73:763-8. http://dx.doi.org/10.1037/0022006X.73.4.763.

32. Karamustafalioglu OK, Zohar J, Guveli M, Gal G, Bakim B, Fostick L, et al. Natural course of posttraumatic stress disorder: a 20-month prospective study of Turkish earthquake survivors. J Clin Psychiatry. 2006;67:882-9. http://dx.doi.org/10.4088/ JCP.v67n0604.

33. Karlehagen S, Malt UF, Hoff H, Tibell E, Herrstromer U, Hildingson $\mathrm{K}$, et al. The effect of major railway accidents on the psychological health of train drivers--II. A longitudinal study of the one-year outcome after the accident. J Psychosom Res. 1993;37:807-17. http://dx.doi.org/10.1016/00223999(93)90170-K.

34. Mayou R, Tyndel S, Bryant B. Long-term outcome of motor vehicle accident injury. Psychosom Med. 1997;59:578-84.

35. Mayou RA, Ehlers A, Bryant B. Posttraumatic stress disorder after motor vehicle accidents: 3-year follow-up of a prospective longitudinal study. Behav Res Ther. 2002;40:66575. http://dx.doi.org/10.1016/S0005-7967(01)00069-9.

36. Mollica RF, Sarajlic N, Chernoff M, Lavelle J, Vukovic IS, Massagli MP. Longitudinal study of psychiatric symptoms, disability, mortality, and emigration among Bosnian refugees. JAMA. 2001;286:546-54. http://dx.doi.org/10.1001/ jama.286.5.546.

37. North CS, Smith EM, Spitznagel EL. One-year followup of survivors of a mass shooting. Am J Psychiatry. 1997;154:1696-702.

38. O’Connor M, Christensen S, Jensen AB, Moller S, Zachariae R. How traumatic is breast cancer? Post-traumatic stress symptoms (PTSS) and risk factors for severe PTSS at 3 and 15 months after surgery in a nationwide cohort of Danish women treated for primary breast cancer. Br J Cancer. 2011;104:419 26. http://dx.doi.org/10.1038/sj.bjc.6606073.

39. O’Donnell ML, Varker T, Creamer M, Fletcher S, McFarlane AC, Silove D, et al. Exploration of DelayedOnset Posttraumatic Stress Disorder After Severe Injury. Psychosom Med. 2013;75:68-75. http://dx.doi.org/10.1097/ PSY.0b013e3182761e 8b.

40. Southwick SM, Morgan CA, III, Darnell A, Bremner D, Nicolaou AL, Nagy LM, et al. Trauma-related symptoms in veterans of Operation Desert Storm: a 2-year follow-up. Am J Psychiatry. 1995;152:1150-5.

41. Stramrood CA, Wessel I, Doornbos B, Aarnoudse JG, van den Berg PP, Schultz WC, et al. Posttraumatic stress disorder following preeclampsia and PPROM: a prospective study with 15 months follow-up. Reprod Sci. 2011;18:645-53. http:// dx.doi.org/10.1177/1933719110395402.

42. Su CY, Tsai KY, Chou FH, Ho WW, Liu R, Lin WK. A three-year follow-up study of the psychosocial predictors of delayed and unresolved post-traumatic stress disorder in Taiwan Chi-Chi earthquake survivors. Psychiatry Clin Neurosci. 2010;64:239-48. http://dx.doi.org/10.1111/j.14401819.2010.02087.x.

43. Sungur M, Kaya B. The onset and longitudinal course of a man-made post-traumatic morbidity: Survivors of the Sivas disaster. International Journal of Psychiatry in Clinical Practice. 2001;5:195-202. http://dx.doi. org/10.1080/136515001317021662.

44. Tjemsland L, Soreide JA, Malt UF. Posttraumatic distress symptoms in operable breast cancer III: status one year after surgery. Breast Cancer Res Treat. 1998;47:141-51. http:// dx.doi.org/10.1023/A:1005957302990.

45. Wadsworth ME, Santiago CD, Einhorn L. Coping with displacement from Hurricane Katrina: predictors of one-year post-traumatic stress and depression symptom trajectories. Anxiety Stress Coping. 2009;22:413-32. http://dx.doi. org/10.1080/10615800902855781.

46. Boscarino JA. Diseases among men 20 years after exposure to severe stress: implications for clinical research and medical care. Psychosom Med. 1997;59:605-14.

47. Bowler RM, Harris M, Li J, Gocheva V, Stellman SD, 
Wilson $\mathrm{K}$, et al. Longitudinal mental health impact among police responders to the $9 / 11$ terrorist attack. Am J Ind Med. 2012;55:297-312. http://dx.doi.org/10.1002/ajim.22000.

48. Cukor J, Wyka K, Mello B, Olden M, Jayasinghe N, Roberts J, et al. The longitudinal course of PTSD among disaster workers deployed to the World Trade Center following the attacks of September 11th. J Trauma Stress. 2011;24:506-14. http:// dx.doi.org/10.1002/jts.20672.

49. Eytan A, Guthmiller A, Durieux-Paillard S, Loutan L, GexFabry M. Mental and physical health of Kosovar Albanians in their place of origin: a post-war 6-year follow-up study. Soc Psychiatry Psychiatr Epidemiol. 2011;46:953-63. http:// dx.doi.org/10.1007/s00127-010-0269-0.

50. Koren D, Arnon I, Klein E. Long term course of chronic posttraumatic stress disorder in traffic accident victims: a three-year prospective follow-up study. Behav Res Ther. 2001;39:1449-58. http://dx.doi.org/10.1016/S00057967(01)00025-0.

51. Scott RB, Brooks N, McKinlay W. Post-traumatic morbidity in a civilian community of litigants: a follow-up at 3 years. J Trauma Stress. 1995;8:403-17. http://dx.doi.org/10.1002/ jts.2490080304.

52. Solomon Z, Mikulincer M. Trajectories of PTSD: a 20-year longitudinal study. Am J Psychiatry. 2006;163:659-66. http:// dx.doi.org/10.1176/appi.ajp.163.4.659.

53. Zhang G, North CS, Narayanan P, Kim YS, Thielman S, Pfefferbaum B. The course of postdisaster psychiatric disorders in directly exposed civilians after the US Embassy bombing in Nairobi, Kenya: a follow-up study. Soc Psychiatry Psychiatr Epidemiol. 2013;48:195-203. http://dx.doi. org/10.1007/s00127-012-0535-4.

54. van der Windt DA, Zeegers MP, Kemper HC, Assendelft WJ, Scholten RJ. [Practice of systematic reviews. VI. Searching, selection and methodological evaluation of etiological research]. Ned Tijdschr Geneeskd. 2000;144:1210-4.

55. Bonzini M, Coggon D, Palmer KT. Risk of prematurity, low birthweight and pre-eclampsia in relation to working hours and physical activities: a systematic review. Occup Environ Med. 2007;64:228-43. http://dx.doi.org/10.1136/ oem.2006.026872.

56. Palmer KT, Bonzini M, Harris EC, Linaker C, Bonde JP. Work activities and risk of prematurity, low birth weight and pre-eclampsia: an updated review with meta-analysis. Occup Environ Med. 2013;70:213-22. http://dx.doi.org/10.1136/ oemed-2012-101032.

57. Bonde JP, Jorgensen KT, Bonzini M, Palmer KT. Miscarriage and occupational activity: a systematic review and metaanalysis regarding shift work, working hours, lifting, standing, and physical workload. Scand J Work Environ Health. 2013;39:325-34. http://dx.doi.org/10.5271/sjweh.3337.

58. Verbeek J, Ruotsalainen J, Hoving JL. Synthesizing study results in a systematic review. Scand J Work Environ Health. 2012;38:282-90. http://dx.doi.org/10.5271/sjweh.3201.
59. Lipsey MW, Wilson DB. Practical meta-analysis. Applied Social Research Methods Series (Vol. 49). Thousand Oaks, CA: Sage Publications, 2001.

60. Borenstein M, Hedges LV, Higgins JP, Rothstein HR. Introduction to meta-analysis. Chichester, UK: Wiley; 2009.

61. Core team. R: A Language and Environment for Statistical Computing 2013. R Foundation for Statistical Computing, Vienna, Austria; 2013. http://www.R-project.org/.

62. Schwarzer G. Meta: Meta-Analysia with R 2013. Package version 3.1-2; 2013. http://CRAN.R-project.org/ package $=$ meta.

63. Viechtbauer W. Conducting meta-analyses in $\mathrm{R}$ with the metafor package. Journal of Statistical Software. 2014;36:148.

64. Boscarino JA, Adams RE. PTSD onset and course following the World Trade Center disaster: findings and implications for future research. Soc Psychiatry Psychiatr Epidemiol. 2009;44:887-98. http://dx.doi.org/10.1007/s00127-0090011-y.

65. van den Berg B, Wong A, van der Velden PG, Boshuizen HC, Grievink L. Disaster exposure as a risk factor for mental health problems, eighteen months, four and ten years post-disaster-a longitudinal study. BMC Psychiatry. 2012;12:147. http:// dx.doi.org/10.1186/1471-244X-12-147.

66. Warner CH, Appenzeller GN, Parker JR, Warner CM, Hoge $\mathrm{CW}$. Effectiveness of mental health screening and coordination of in-theater care prior to deployment to Iraq: a cohort study. Am J Psychiatry. 2011;168:378-85. http://dx.doi. org/10.1176/appi.ajp.2010.10091303.

67. Brauer C, Mikkelsen S. The context of a study influences the reporting of symptoms. Int Arch Occup Environ Health. 2003;76:621-4. http://dx.doi.org/10.1007/s00420-003-04634.

68. LaGuardia RL, Smith G, Francois R, Bachman L. Incidence of delayed stress disorder among Vietnam era veterans: the effect of priming on response set. Am J Orthopsychiatry. 1983;53:18-26. http://dx.doi.org/10.1111/j.1939-0025.1983. tb03345.x.

69. Ramchandani D. Distinguishing features of delayedonset posttraumatic stress disorder. Bull Menninger Clin. 1990;54:247-54

70. Pomerantz AS. Delayed onset of PTSD: delayed recognition or latent disorder? Am J Psychiatry. 1991;148:1609.

71. Herrmann N, Eryavec G. Delayed onset post-traumatic stress disorder in World War II veterans. Can J Psychiatry. 1994;39:439-41.

72. Iversen AC, van SL, Hughes JH, Browne T, Greenberg N, Hotopf M, et al. Help-seeking and receipt of treatment among UK service personnel. Br J Psychiatry. 2010;197:149-55. http://dx.doi.org/10.1192/bjp.bp.109.075762.

Received for publication: 25 September 2013 